196

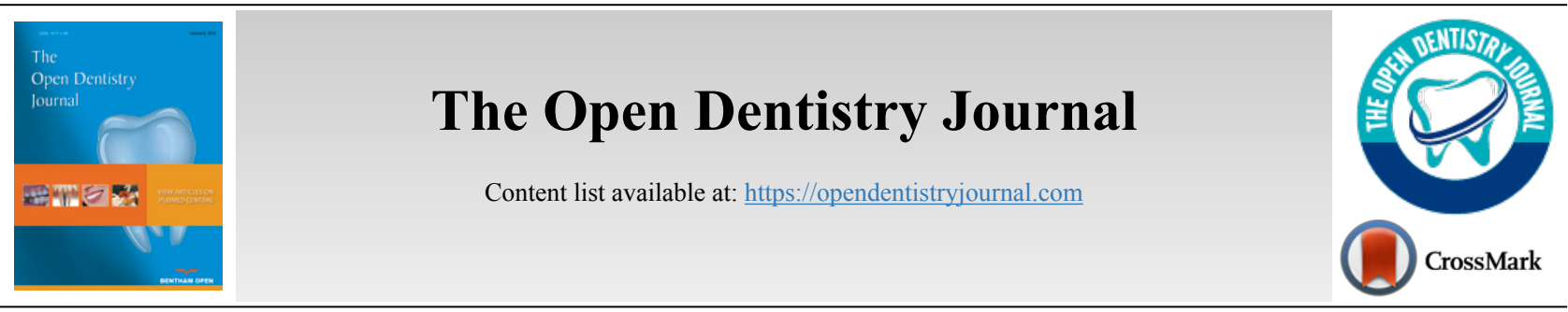

RESEARCH ARTICLE

\title{
Comparison of the Expression of Periodontal Markers in Dental and Bone Marrow-derived Mesenchymal Stem Cells.
}

\author{
Devy Garna $^{1,2(\mathbb{C})}$, Manmeet $\operatorname{Kaur}^{1}(\mathbb{O})$, Francis J Hughes ${ }^{1, *(\infty)}$ and Mandeep Ghuman ${ }^{1}$ \\ ${ }^{1}$ Centre for Host-Microbial Interactions, Faculty of Oral, Dental and Craniofacial Sciences, King's College London, London, UK \\ ${ }^{2}$ Department of Periodontology, Faculty of Dentistry, Padjadjaran University, Sumedang , Indonesia
}

\begin{abstract}
:
Background:

Periodontal ligament stem cells are a source of mesenchymal stem cells, but it is unclear whether their phenotype is distinct from mesenchymal stem cells derived from different tissues, such as those derived from bone marrow.

Objective:

To investigate the expression of the putative PDL markers asporin, periostin, nestin and cementum protein 1 , by periodontal ligament stem cells both constitutively and during osteogenic differentiation when compared to bone marrow-derived mesenchymal stem cells, and dental pulp stem cells.

\section{Methods:}

The primary human periodontal ligament, bone marrow, and dental pulp stem cells, and osteoblasts from different donors were cultured in vitro. The expression of periodontal marker associated genes during osteogenic induction was tested by qRT-PCR and immunofluorescence staining.

Results:

Asporin expression was detected in periodontal ligament stem cells and increased markedly during the time in culture (upregulated x 53 fold at 21 days post-induction). During osteogenic differentiation, asporin expression significantly decreased in periodontal ligament cells whereas periostin significantly decreased in dental pulp cells. Periostin expression was absent in osteoblasts, but expression gradually increased in all other cells with time in culture. Nestin expression was mainly seen in the periodontal ligament and dental pulp cells and was largely absent in osteoblasts and bone marrow cells. Cementum protein-1 was most highly expressed in bone marrow cells and osteoblasts following osteogenic induction.

\section{Conclusions:}

The results provide further evidence that periodontal ligament-derived and bone marrow derived mesenchymal stem cells are phenotypically distinct. Periodontal markers are also expressed in dental pulp stem cells.
\end{abstract}

Key words: Periodontal, Mesenchymal stem cells, Asporin, Periostin, Cementum Protein-1, Bone marrow.

\begin{tabular}{l|l|l|l} 
Article History & Received: January 21, 2020 & Revised: March 17, 2020 & Accepted: March 17, 2020
\end{tabular}

\section{INTRODUCTION}

Mesenchymal Stem Cells (MSCs) are adult stem cells that exhibit self-renewal and have the capacity to differentiate into osteoblast, chondrocyte, and adipocytic lineages. They were originally isolated from the stroma of bone marrow [1] but since then have also been isolated from many other tissues.

\footnotetext{
* Address correspondence to this author at the Centre for Host-Microbial Interactions, Faculty of Oral, Dental and Craniofacial Sciences, King's College London, Floor 17 Tower Wing, Guys Hospital, London SE1 9RT, UK;

Tel: +44 (0)20 7848 8478; E-mail: francis.hughes@kcl.ac.uk
}

Within the dental tissues MSCs have been isolated from Periodontal Ligament (PDL), dental pulp, dental follicle, exfoliated teeth (SHED cells) and gingival connective tissues [2].

There is considerable interest in the potential therapeutic application of MSCs for tissue regeneration, including for periodontal regeneration. A number of animal studies have demonstrated the principle of periodontal regeneration using MSCs derived from sources including bone marrow, PDL, gingiva, dental pulp and adipose tissue [3 - 8]. However, the 
question remains whether these different MSC sources are phenotypically distinct, which might render them particularly suitable for a specific regenerative application such as periodontal regeneration. A few studies have suggested that PDL-derived MSC may have phenotypic differences with other MSCs [9 - 11]. Studies to address this question are hampered by the absence of any specific markers for either PDL cells or tissues. Nevertheless, a number of proteins are reported to be highly expressed in PDL and have been proposed as PDL markers in studies investigating differences between MSC phenotypes, even though they may not be uniquely expressed in that tissue only. These putative candidate PDL markers include periostin, asporin (periodontal ligament associated protein-1, PLAP-1), cementum protein-1 (CEMP-1) and the neural crest-derived tissue marker Nestin [12].

Asporin is a member of the small leucine-rich proteoglycan family and was originally described as being localised to the periodontal ligament, although it is expressed in a number of other tissues [13].

Periostin is a matricellular protein involved with the maintenance of connective tissue integrity which is known to be highly expressed in the periodontal ligament, as well as in the connective tissues including bone.

Nestin is an Intermediate Filament (IF) protein, which interacts with other IF proteins in the cytoskeleton. Nestin expression is characteristic of neural crest-derived cells, particularly when undifferentiated [14, 15].

Cementum-protein-1 (CEMP-1) was first isolated from a cementoblastoma as a potentially cementum-specific protein. CEMP-1 expression is seen in cementoblasts but has also been reported in bone [16]. CEMP-1 reportedly has growth factorlike activity on PDL cells and other undifferentiated mesenchymal cells stimulating osteoblast/cementoblast differentiation.

Consequently, the aim of the study here is to test the hypothesis that PDL-derived MSC are phenotypically distinct from MSCs derived from other sources. Specifically, the aim was to test the hypothesis that PDL-derived MSC (PDLSC) will differentially express high levels of periostin, asporin, CEMP-1, and nestin when compared to expression in dental pulp-derived MSC (DPSC) and bone marrow-derived MSCs (BMSCs).

\section{MATERIALS AND METHODS}

\subsection{Overview}

PDLSC and BMSC derived from 3 different donors along with DPSC from 2 different donors were tested for the expression of marker genes. Primary osteoblast cultures were also tested as control cultures. Cells were first tested for their MSC properties by the capacity to undergo multilineage differentiation and expression of MSC-associated cell surface markers. Gene expression of aspirin (ASPN), periostin (POSTN), nestin (NES) and cementum protein-1 (CEMP1) were tested in cell lines at time points up to 21 days, in normal culture medium and osteogenic medium. Protein expression was confirmed by immunofluorescence.

\subsection{Cell Culture}

Periodontal ligament cell cultures were established from explants derived from healthy third molar teeth extracted from three patients aged between 20-35 years old (Arceo 1991). Ethical approval for obtaining the samples was obtained from Kent NHS Research Ethics Committee (Reference No: 11/LO/0259). Primary human dental pulp stem cell cultures (DPSC) and human osteoblast cultures derived from alveolar bone and femur were kindly provided by Professor Lucy DiSilvio, King's College London. Primary human bone marrow stromal MSCs (BMSC) were purchased commercially (Lonza, Slough, UK). The detailed phenotypic features of the three specific BMSC lines have been described extensively in a previous publication from our group [17]. Cells were maintained in $\alpha$-Minimal Essential Medium (MEM), penicillin $50 \mathrm{U} / \mathrm{ml}$, streptomycin $50 \mu \mathrm{g} / \mathrm{ml}$ (Sigma-Aldrich), Glutamax 2 $\mathrm{mM}$ (Invitrogen, Paisley, UK), and $10 \%$ fetal bovine serum/FBS (Sigma-Aldrich) (normal growth medium) at $37^{\circ} \mathrm{C}$ in a humidified $5 \% \mathrm{CO}_{2}, 95 \%$ air atmosphere. For experiments involving the stimulation of osteogenic differentiation, normal growth medium was supplemented additionally with $0.1 \mu \mathrm{M}$ dexamethasone, $0.05 \mathrm{mM}$ ascorbic acid, and $10 \mathrm{mM} \beta$ glycerophosphate (Sigma-Aldrich). For adipocytic differentiation, normal growth medium was supplemented with $1 \mu \mathrm{M}$ dexamethasone, $0.25 \mathrm{mM}$ isobutylmethylxanthine, $50 \mu \mathrm{M}$ indomethacin and $10 \mu \mathrm{g} / \mathrm{ml}$ insulin (Sigma-Aldrich) and for chondrocytic differentiation with $1 \mu \mathrm{M}$ dexamethasone, $1 \mu \mathrm{M}$ ascorbic acid, 1\% sodium pyruvate (Invitrogen) and 10ng/ml transforming growth factor-beta 1 (TGF- $\beta 1$, Peptrotech, Rocky Hill, NJ, USA).

All cell types were used in the same passage in each experiment (passage 3-7). Differentiation assays were carried out as previously described [17].

\subsection{Flow Cytometry}

MSC surface marker expression was measured using fourcolour flow cytometry analysis, with the FACSCanto II flow cytometer (BD Biosciences, San Jose, CA). Briefly, 100,000 cells were incubated with Fc block (BioLegend, Cambridge, U.K.) in $50 \mu 1$ of fluorescence-activated cell sorting (FACS) buffer for 15 minutes at room temperature. The antibody cocktail was divided into two groups (A\&B). Group A contained CD34-PerCP/Cy5.5 (clone 4H11), CD105fluorescein isothiocyanate (FITC) (clone 43A3), CD146phycoerythrin, and (PE)/Cy7 (clone SHM-57) whereas group B contained CD45-PerCP/Cy5.5 (clone HI30), CD44-FITC (clone BJ18), and CD90-allophycocyanin (APC) (clone 5E10). Adding an antibody cocktail into the FACS tube, which contained the cells suspension, promoted direct binding.

Unstained cells, compensations beads, and fluorescence minus one (FMO) were used to set the gating. All antibodies were obtained from Bio-Legend U.K. (Cambridge Bioscience) and used at the recommended concentration. Cells were incubated for 15 minutes at room temperature in the dark. After being washed twice, cells were resuspended in $300 \mu$ of FACS buffer and immediately being analysed. A total of 10,000 events were acquired for each sample and data analysis was performed using DIVA software (BD Biosciences). 


\subsection{Real Time PCR}

Total RNA was extracted using TRI reagent (Ambion, Warrington, UK). Cells in 12-well plates were lysed in $600 \mathrm{ml}$ TRI reagent and transferred into Phase Lock Gel Heavy tubes (5 prime, VWR, Leicestershire, UK) according to the manufacturer's instructions. RNA purity and quantity were assessed by Nano Drop 1000 Mass spectrometer (Fisher Scientific). Ratios A260/A280 between 1.8-2 were considered to be high purity and used for cDNA synthesis. Possible DNA contamination was removed and cDNA prepared from $1 \mu \mathrm{g}$ RNA using QuantiTect Reverse Transcription Kit (Qiagen, West Sussex, UK) according to manufacturer's instructions. qRT-PCR was performed with a Rotor-Gene 6000 thermal cycler (Qiagen) using Brilliant III Ultra-Fast SYBR Green qPCR Master mix (Stratagene, Agilent Technologies, Cheshire, UK). Primer pair sequences are listed in Table 1. PCR conditions consisted of 1 cycle of $95^{\circ} \mathrm{C}$ for $3 \mathrm{~min}, 40$ cycles of $95^{\circ} \mathrm{C}$ for $10 \mathrm{sec}, 60^{\circ} \mathrm{C}$ for $10 \mathrm{sec}$, and followed by melting analysis of 1 cycle with gradual increase from $65^{\circ} \mathrm{C}$ to $95^{\circ} \mathrm{C}$. RPL13a was used as the reference gene

Table 1. List of PCR Primers used

\begin{tabular}{|c|c|c|c|}
\hline Gene & Gene Acc & Forward 5'-3' & Reverse 5'-3' \\
\hline RPL13a & NM & CATCGTGGCTAAACAGGTACTG & GCACGACCTTGAGGGCAGCC \\
& 000977 & & \\
\hline ASPN & SY & CCCTTCAGGATTACCAGAGTTG & TTGGCACTGTTGGACAGAAG \\
& 130414037 & & \\
\hline CEMP1 & SY & CCTGGGGATGTGTGAAGAGA & TGTTTTCTGCTCCCTGGACT \\
& 140609461 & & \\
\hline NES & SY & CTCCAAGACTTCCCTCAGCT & TCAGGACTGGGAGCAAAGAT \\
& 140307726 & & \\
\hline POSTN & SY & TGACACAACCTGGAGACTGG & GAGCATTTTTGTCCCGTATCA \\
& 130414039 & & \\
\hline
\end{tabular}

\subsection{Analysis of qRT PCR Data}

To calculate the relative expression of genes, all samples were normalized to their housekeeping gene (RPL) cycle threshold $(\mathrm{Ct})$ value and then normalised to the average of PDLSC Ct value at day 0 (24 hours after seeding the cells). Thus normalised data from each different cell line (of the same derivation) were combined for analyses. The fold change in gene expression was assessed using the comparative method. Data were expressed as mean \pm S.D. Either one-way, or twoway, ANOVA with Bonferroni post test was used to determine whether differences in gene expression levels in the different cell types were statistically significant from control. Statistical analysis was carried out using Prism 5 v5.0 software (Graph Pad Software Inc.). The statistical significance was set at $\mathrm{p}<0.05$.

\subsection{Immunofluorescence Staining}

Cells were seeded in normal media and at day 3 cells were fixed for 5 minutes with $100 \%$ methanol for asporin or periostin staining, and 10 minutes $4 \%$ formaldehyde fixed for nestin staining. Thereafter the cells were incubated in $1 \% \mathrm{BSA} /$ $10 \%$ normal goat serum $/ 0.3 \mathrm{M}$ glycine in $0.1 \%$ PBS-Tween for 1 hour to permeabilise the cells and block non-specific protein-protein interactions. The cells were then incubated with the antibody asporin $(\mathrm{ab} 58741,5 \mu \mathrm{g} / \mathrm{ml})$, periostin (ab14041, $1 / 1000$ dilution) and nestin (ab 105389,1/200 dilution) overnight at $+4^{\circ} \mathrm{C}$. The primary antibody was visualized using Alexa Fluor ${ }^{\circledR}$ 555-conjugated secondary antibodies anti-rabbit $\operatorname{IgG}(\mathrm{H}+\mathrm{L}), \mathrm{F}(\mathrm{ab}$ '2) (Cell Signaling \#4413) at a 1/250 dilution for 1 hour. DAPI was used to counterstain the cell nuclei (blue) at a concentration of $1.43 \mu \mathrm{M}$. As negative controls, some samples were incubated without the primary antibody. Analysis particle from ImageJ was used to determine the integrated density as a semi-quantitative estimate of protein expression corrected for cell number. This program measures the cell numbers from the DAPI nuclear counter stain, and the total positive fluorescence area in each image.

\section{RESULTS}

\subsection{Stem Cell Characteristics of Cells}

Flow cytometry demonstrated that a very high proportion of PDLSC and BMSC cells expressed CD44, CD90, CD146, $\mathrm{CD} 105$, and CD45 and did not express the hematopoietic cell markers CD34 and CD45. DPSCs also showed a similar expression pattern of surface markers except that CD146 was only expressed in $72 \%$ of cells, and CD105 expression was particularly low with only $11 \%$ of cells (Table 2 ). (NB the data for BMSC shown here was obtained in a previously published study from our group [17].

Table 2. Percentage of cells expressing cell surface markers (mean \pm SD).

\begin{tabular}{|c|c|c|c|c|c|c|c|}
\hline- & $\mathbf{N}$ & CD44 & CD146 & CD90 & CD105 & CD45 & CD34 \\
\hline & $99.3 \% \pm$ & $95.4 \% \pm$ & $99.4 \% \pm$ & $86.4 \%+$ & $0.8 \% \pm$ & $0.7 \%$ \\
PDLSC & 3 & 1.1 & 3.9 & 0.7 & 10.9 & 0.8 & +0.9 \\
\hline & & $99.8 \% \pm$ & $72.3 \% \pm$ & $97.1 \%+$ & $11.7 \%+$ & $0.5 \% \pm$ & $0.5 \% \pm$ \\
DPSC & 2 & 0.1 & 15.1 & 0.2 & 2.1 & 0.7 & 0.2 \\
\hline & & $95.1 \% \pm$ & $91.8 \% \pm$ & $96.7 \% \pm$ & $95.6 \% \pm$ & $1.2 \% \pm$ & $2.0 \% \pm$ \\
BMSC & 3 & 4.4 & 4.6 & 6.4 & 3.8 & 1.2 & 0.7 \\
\hline
\end{tabular}

PDLSCs, DPSCs, and BMSCs were able to differentiate into osteoblast, adipocyte, and chondrocyte lineages following 21 days in osteogenic, adipogenic or chondrogenic culture medium respectively as demonstrated by the formation of alizarin-red staining matrix, alcian blue staining or Oil-Red-O staining intracellular droplets. There were no significant differences in cell growth rates between different cell types (data not shown).

\subsection{Gene Expression of Putative Periodontal Markers in vitro.}

\subsubsection{Asporin}

Asporin expression was seen mainly in PDLSC and, to a lesser extent, in DPSC. For cells grown in both normal and osteogenic medium, asporin expression in PDLSCs increased by around 50-fold over 21 days (Fig. 1A, 2A). DPSC also showed an increased expression of asporin with time but this was significantly less than in PDLSC at all timepoints. There were consistently low levels of expression of asporin by BMSCs and osteoblasts at all time points in either culture condition (Fig. 1A, 2A). 
A

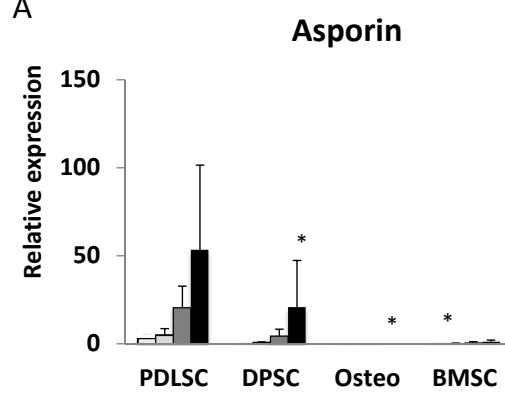

B

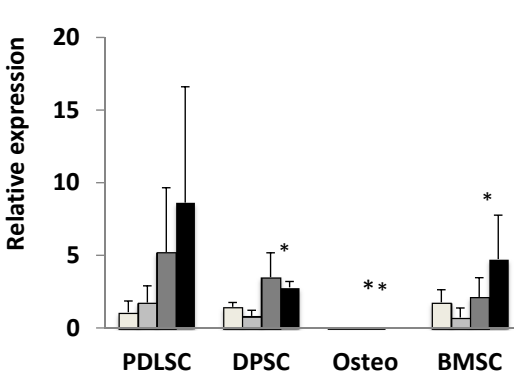

C

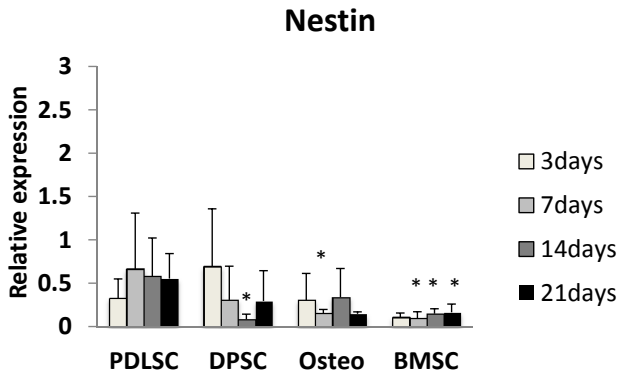

D

CEMP-1

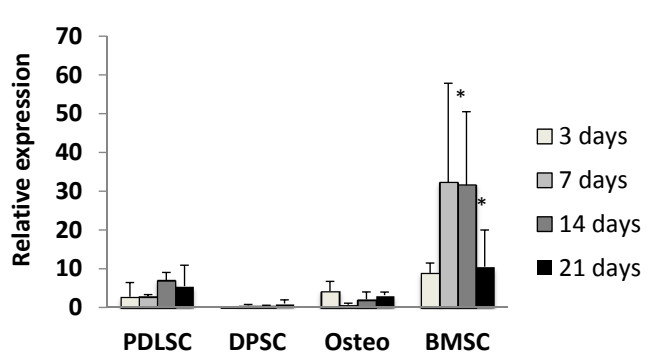

Fig. (1). Gene expression of cells in normal medium determined by qRT PCR. Each cell type tested consisted of 3 lines derived by different donors (2 lines with DPSC) and each line was carried out in triplicate. Mean \pm SD. Differences were analysed by 2-way ANOVA and significant differences tested by Bonferoni post test. * Significantly different from PDL at that time point P $<0.01$. A) Asporin (ASPN); B) Periostin (POSTN); C) Nestin (NES) ; D) Cementum Protein-1 (CEMP1).

A

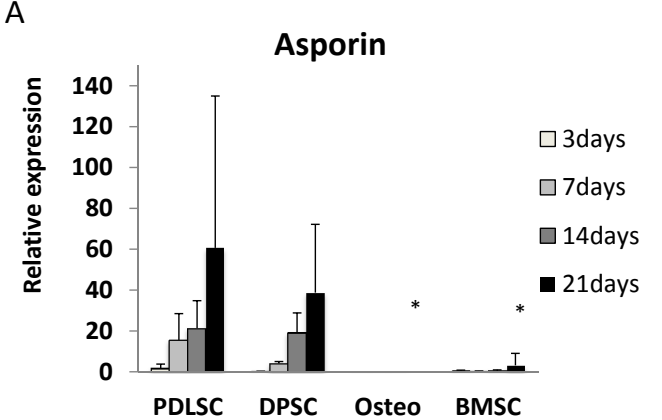

B

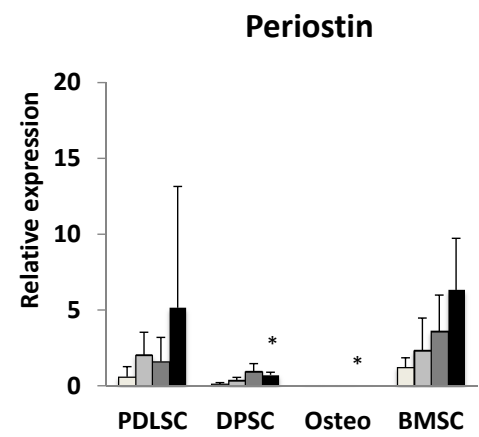

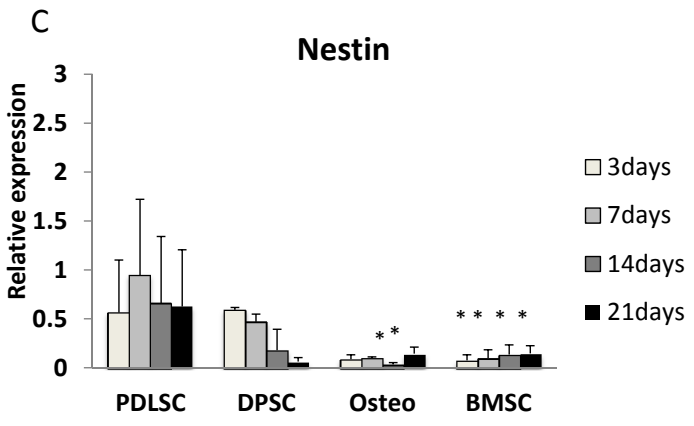

D

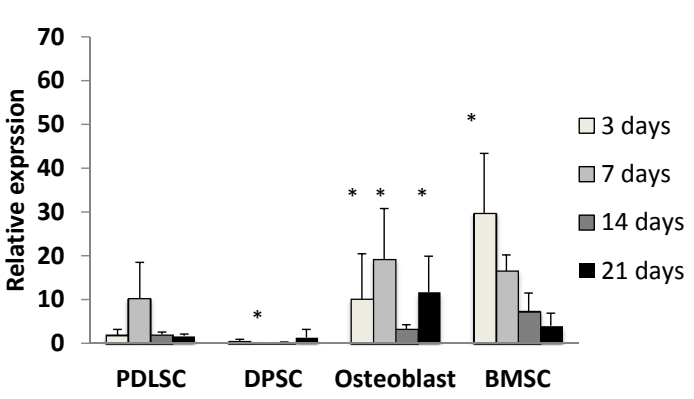

Fig. (2). Gene expression of cells in osteogenic medium determined by qRT PCR. Each cell type tested consisted of 3 lines derived by different donors (2 lines with DPSC) and each line was carried out in triplicate. Mean \pm SD. Differences were analysed by 2-way ANOVA and significant differences tested by Bonferoni post test. *Significantly different from PDL at that time point $\mathrm{P}<0.01$. A) Asporin (ASPN); B) Periostin (POSTN); C) Nestin (NES) ; D) Cementum Protein-1 (CEMP1). 


\subsubsection{Periostin}

Periostin expression was seen mainly in PDLSCs and BMSCs. In normal medium periostin expression was significantly increased in PDLSC by day 21, when compared to that seen in other cell types (Fig. 1B). In cultures with osteogenic medium, periostin expression in PDLSCs and BMSCs was similar and increased significantly with time in culture (Fig. 2B). There was no detectable expression of periostin in osteoblasts. In addition, periostin expression was lower in all cell types cultured in osteogenic media compared to those in normal media (Fig. 2B).

\subsubsection{Nestin}

Nestin expression was reduced at all time points when compared to the baseline (day 0). The expression of nestin was higher in PDLSCs and DPSCs when compared to BMSC and osteoblasts in both normal and osteogenic media (Fig. 1C, 2C).

\subsubsection{CEMP-1}

In normal medium, the highest levels of expression were seen in BMSCs, which reached its maximum at days 7 and 14
(Fig. 1D). In contrast expression of CEMP-1 was consistently low in PDLSC, DPSC and osteoblasts. In osteogenic medium, the greatest expression of CEMP-1 in BMSC was seen at day 3 (Fig. 2D) and declined at later time points. In addition, increased CEMP-1 expression was also seen in osteoblast cultures in osteogenic medium (Fig. 2D).

\subsubsection{Expression of periodontal ligament associated proteins.}

Immunofluorescence cytochemistry was used to test for protein expression. The results of immunofluorescence studies for asporin, periostin, and nestin are shown in Fig. (3). Positive staining for all 3 proteins was seen particularly in PDLSC, although nestin staining was also particularly high in DPSC. Immunofluorescence staining was not carried out for CEMP-1 as there was no significant expression of CEMP-1 seen in PDLSC at the mRNA level.

Quantification of integrated fluorescence intensity, which corrects for different numbers of cells between fields, is shown in Fig. (3B). Results demonstrate significantly higher expression of asporin, periostin, and nestin in PDLSC compared to BMSC. Staining in osteoblasts was almost nonexistent throughout.
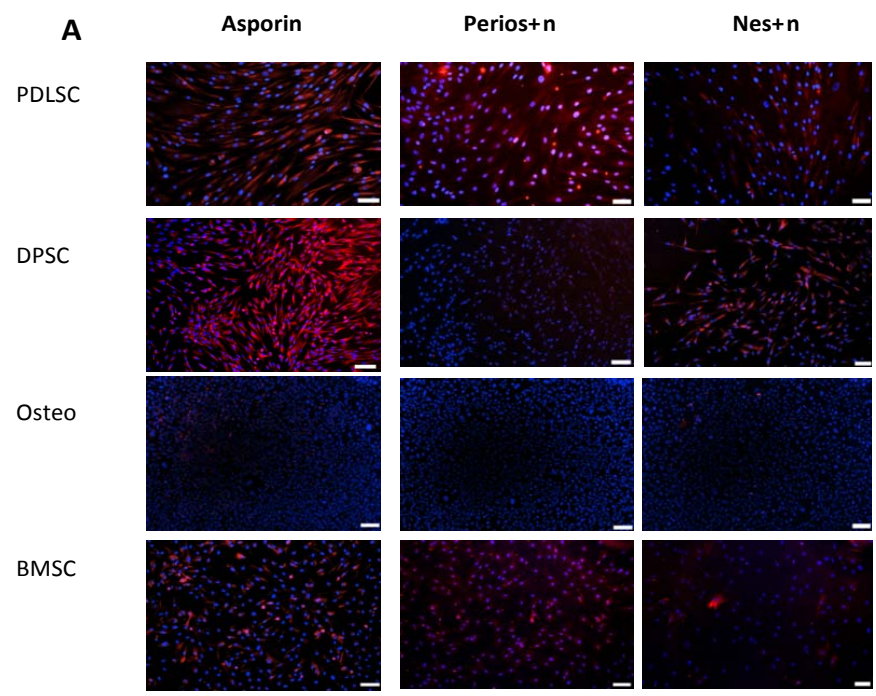

B
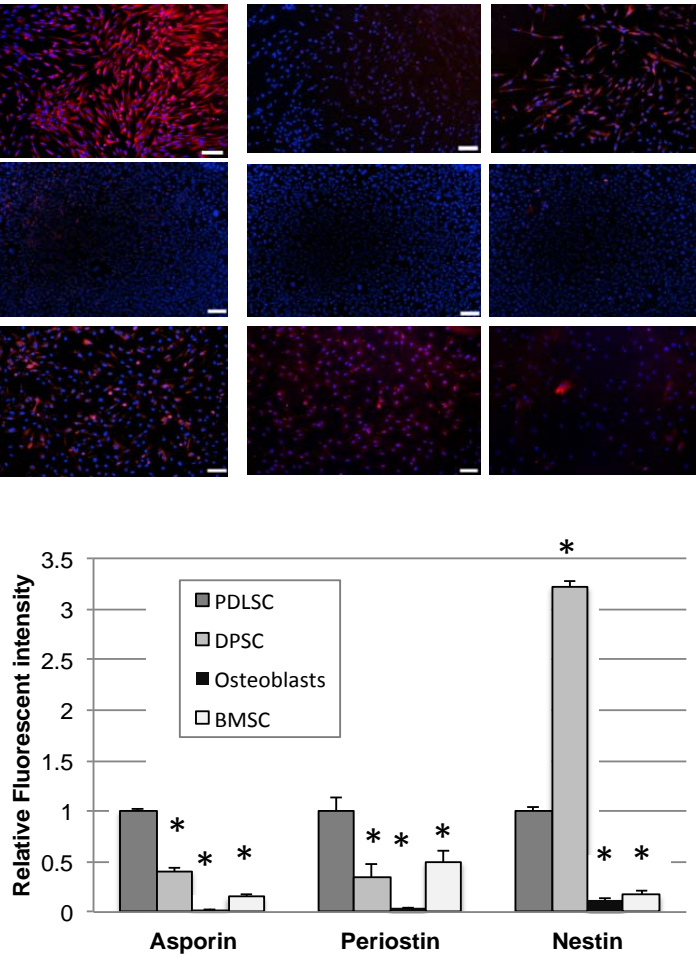

Fig. (3). A) Immunofluorescent staining of cells for asporin, periostin and nestin. PDLSC: Periodontal ligament stem cells, DPSC dental pulp-derived stem cells, BMSC bone marrow derived mesenchymal stem cells. B) Relative fluorescent intensity results from immunofluorescent studies normalised for cell numbers. Mean \pm SD. 3 cultures for each cell type tested. * Significantly different from PDLSC, P $<0.05,1$-way ANOVA and Bonferoni post test. 


\section{DISCUSSION}

Phenotypic differences between MSCs derived from different tissues have been proposed as potentially of importance in selecting cell sources for optimal tissue engineering strategies. Some studies have tried to address this issue in dental stem cells and there is at least some evidence from non-human sources that there are quantitative (but not qualitative) differences in protein expression patterns between PDLSCs, BMSCs and DPSCs $[18,19]$.

We postulated that potential candidate genes to distinguish between PDLSC and other MSCs might reasonably be those of proteins that are highly expressed preferentially in the periodontal ligament, including asporin, periostin, nestin and cementum protein-1 (CEMP-1). This required that high expression levels of these proteins may occur constitutively in cells associated with the periodontal ligament, rather than be dependent on environmental cues present within the specific tissue itself.

The results of the studies described here firstly support the idea that asporin, periostin, and nestin are constitutively highly expressed in PDLSC, in line with previous studies demonstrating that they are abundant in the periodontal ligament. The $4^{\text {th }}$ candidate marker that was investigated, CEMP-1, in contrast, was not associated with PDLSC but was constitutively expressed by BMSC. Interestingly, when cells were exposed to osteogenic medium, osteoblast cultures also showed CEMP-1 expression, but this was not seen with PDLSC or with DPSC. Although the studies carried out here were based largely on gene expression data, our studies of protein expression by immunofluorescence are largely in line with the findings at the mRNA level. However, we did not carry out a full series of experiments at later time points where some of the changes in gene expression are most evident, and quantification of immunofluorescence intensity is, at best, a semi-quantitative method of assessing protein expression.

The results suggest that these MSC lines isolated from different tissues show inherent differences in constitutive gene expression. PDLSC expressed high levels of asporin, periostin and nestin but not CEMP-1. In contrast, BMSC showed low levels of asporin and nestin expression but did express periostin and also expressed CEMP-1. DPSC expressed asporin and nestin, and to a lesser extent periostin, but not CEMP-1. Thus, each MSC cell type had a distinct pattern of expression of the candidate genes tested.

Ideally it would be helpful to test MSC cell phenotype differences in cells that were all derived from the same donor. However, unfortunately this was not feasible due to issues of tissue availability from donors. However, as we were able to test cells from 3 different donors for BMSC and PDLSC (and 2 from DPSC) and combine the data from these, our results show that differences in expression of these genes were more associated with differences in the source of tissue derivation than with differences between donors.

Although asporin expression is localised to the periodontal ligament, it has been shown to be expressed in a number of tissues, most notably in cartilage and bone, and is able to attach to collagen fibres and bind calcium [13]. Therefore, its expression in PDLSC was predicted, although the absence of expression in BMSCs, even after stimulation of osteogenic differentiation was perhaps less expected. Similarly, periostin has also been reported to be expressed in bone as well as in periodontal ligament. Thus, our results of periostin expression in both PDLSC and BMSC are consistent with these previous findings.

Nestin expression is considered a marker of neural crestderived cells. Therefore, our results support these previous findings with high expression of nestin seen in the neural crestderived PDLSC and DPSC, but not in BMSC or the osteoblast control cultures.

As already discussed, CEMP-1 expression has been described in the bone as well as in cementum. Thus, it was not unexpected to see CEMP-1 gene expression in BMSC, but much less expected that PDLSC did not express CEMP-1, even after 21 days of stimulation in osteogenic medium and despite the fact that these specific cell cultures are known to undergo osteoblastic differentiation and expression of osteoblast associated genes during culture in osteogenic medium as used here.

Overall the differences in expression patterns of these putative periodontal markers support the hypothesis of there being constitutive differences in mesenchymal stem cell populations derived from periodontal, dental pulp and bone marrow sources, which persist even following osteoblastic induction. The functional significance of these differences is currently uncertain. One clinically relevant issue is whether PDLSC may be particularly suited for application in periodontal regenerative tissue engineering procedures. Despite this possibility, mesenchymal stem cells from a range of different sources have all been reported to be able to contribute to the formation of new periodontal attachment in preclinical animal studies, and do not provide any evidence either way of the relative clinical effectiveness of MSC from difference sources.

\section{CONCLUSION}

There were clear differences in expression patterns between PDLSCs and BMSCs, which are suggestive of innate phenotypic differences between MSC types. PDLSC, in particular, expressed high levels of the matricellular proteins periostin and asporin, and the neural crest-derived cytoskeletal protein nestin. Gene expression of Cementum Protein-1 was mainly seen in BMSC and osteoblasts and not in PDLSC.

\section{ETHICS APPROVAL AND CONSENT TO PARTI- CIPATE}

Ethical approval for obtaining the tissue samples was obtained from Kent NHS Research Ethics Committee, UK (Reference No: 11/LO/0259).

\section{HUMAN AND ANIMAL RIGHTS}

No animals were used in this research. All human research procedures followed were in accordance with the ethical standards of the committee responsible for human experimentation (institutional and national), and with the Helsinki Declaration of 1975, as revised in 2013. 


\section{CONSENT FOR PUBLICATION}

Written informed consent was obtained from each participant prior to the study.

\section{AVAILABILITY OF DATA AND MATERIALS}

The data that support the findings of this study are available from the corresponding author, [F.H], upon reasonable request.

\section{FUNDING}

DG is generously supported by the Directorate General of Higher Education Indonesia Ministry of Education. Grant number 373.33/E4.4/K/2012.

\section{CONFLICTS OF INTEREST}

The authors report no conflict of interest associated with this work.

\section{ACKNOWLEDGEMENTS}

Declared none.

\section{REFERENCES}

[1] Friedenstein AJ, Deriglasova UF, Kulagina NN, et al. Precursors for fibroblasts in different populations of hematopoietic cells as detected by the In vitro. colony assay method. Exp Hematol 1974; 2(2): 83-92. [PMID: 4455512]

[2] Hughes FJ. Mesenchymal Stem Cells and Periodontal Regeneration. Curr Oral Health Rep 2014; 1: 1-8.

[http://dx.doi.org/10.1007/s40496-013-0010-7]

[3] Seo BM, Miura M, Gronthos S, et al. Investigation of multipotent postnatal stem cells from human periodontal ligament. Lancet 2004; 364(9429): 149-55.

[http://dx.doi.org/10.1016/S0140-6736(04)16627-0] [PMID: 15246727]

[4] Fawzy El-Sayed KM, Paris S, Becker ST, et al. Periodontal regeneration employing gingival margin-derived stem/progenitor cells: an animal study. J Clin Periodontol 2012; 39(9): 861-70.

[http://dx.doi.org/10.1111/j.1600-051X.2012.01904.x] [PMID: 22694281]

[5] Han J, Menicanin D, Marino V, et al. Assessment of the regenerative potential of allogeneic periodontal ligament stem cells in a rodent periodontal defect model. J Periodontal Res 2013. [PMID: 23841948]

[6] Mrozik KM, Wada N, Marino V, et al. Regeneration of periodontal tissues using allogeneic periodontal ligament stem cells in an ovine model. Regen Med 2013; 8(6): 711-23.

[http://dx.doi.org/10.2217/rme.13.66] [PMID: 24147527]

[7] Tobita M, Uysal AC, Ogawa R, Hyakusoku H, Mizuno H. Periodontal tissue regeneration with adipose-derived stem cells. Tissue Eng Part A 2008; 14(6): 945-53.

[http://dx.doi.org/10.1089/ten.tea.2007.0048] [PMID: 18558814]

[8] Yang H, Gao LN, An Y, et al. Comparison of mesenchymal stem cells derived from gingival tissue and periodontal ligament in different incubation conditions. Biomaterials 2013; 34(29): 7033-47.

[http://dx.doi.org/10.1016/j.biomaterials.2013.05.025] [PMID: 23768902]

[9] Tamaki Y, Nakahara T, Ishikawa H, Sato S. In vitro. analysis of mesenchymal stem cells derived from human teeth and bone marrow. Odontology 2013; 101(2): 121-32.

[http://dx.doi.org/10.1007/s10266-012-0075-0] [PMID: 22772774]

[10] Huang GT, Gronthos S, Shi S. Mesenchymal stem cells derived from dental tissues $v s$. those from other sources: their biology and role in regenerative medicine. J Dent Res 2009; 88(9): 792-806.

[http://dx.doi.org/10.1177/0022034509340867] [PMID: 19767575]

[11] Tsumanuma Y, Iwata T, Washio K, et al. Comparison of different tissue-derived stem cell sheets for periodontal regeneration in a canine 1-wall defect model. Biomaterials 2011; 32(25): 5819-25.

[http://dx.doi.org/10.1016/j.biomaterials.2011.04.071] [PMID: 21605900]

[12] Marchesan JT, Scanlon CS, Soehren S, Matsuo M, Kapila YL. Implications of cultured periodontal ligament cells for the clinical and experimental setting: a review. Arch Oral Biol 2011; 56(10): 933-43. [http://dx.doi.org/10.1016/j.archoralbio.2011.03.003] [PMID: 21470594]

[13] Yamada S, Murakami S, Matoba R, et al. Expression profile of active genes in human periodontal ligament and isolation of PLAP-1, a novel SLRP family gene. Gene 2001; 275(2): 279-86.

[http://dx.doi.org/10.1016/S0378-1119(01)00683-7] [PMID: 11587855]

[14] Wiese C, Rolletschek A, Kania G, et al. Nestin expression--a property of multi-lineage progenitor cells? Cell Mol Life Sci 2004; 61(19-20): 2510-22.

[http://dx.doi.org/10.1007/s00018-004-4144-6] [PMID: 15526158]

[15] Gronthos S, Brahim J, Li W, et al. Stem cell properties of human dental pulp stem cells. J Dent Res 2002; 81(8): 531-5.

[http://dx.doi.org/10.1177/154405910208100806] [PMID: 12147742]

[16] Alvarez-Pérez MA, Narayanan S, Zeichner-David M, Rodríguez Carmona B, Arzate H. Molecular cloning, expression and immunolocalization of a novel human cementum-derived protein (CP-23). Bone 2006; 38(3): 409-19.

[http://dx.doi.org/10.1016/j.bone.2005.09.009] [PMID: 16263347]

[17] Gharibi B, Hughes FJ. Effects of medium supplements on proliferation, differentiation potential, and In vitro. expansion of mesenchymal stem cells. Stem Cells Transl Med 2012; 1(11): 771-82. [http://dx.doi.org/10.5966/sctm.2010-0031] [PMID: 23197689]

[18] Mrozik KM, Zilm PS, Bagley CJ, et al. Proteomic characterization of mesenchymal stem cell-like populations derived from ovine periodontal ligament, dental pulp, and bone marrow: analysis of differentially expressed proteins. Stem Cells Dev 2010; 19(10): 1485-99.

[http://dx.doi.org/10.1089/scd.2009.0446] [PMID: 20050811]

[19] Eleuterio E, Trubiani O, Sulpizio M, et al. Proteome of human stem cells from periodontal ligament and dental pulp. PLoS One 2013; $8(8) \mathrm{e} 71101$

[http://dx.doi.org/10.1371/journal.pone.0071101] [PMID: 23940696]

(C) 2020 Garna et al.

This is an open access article distributed under the terms of the Creative Commons Attribution 4.0 International Public License (CC-BY 4.0), a copy of which is available at: (https://creativecommons.org/licenses/by/4.0/legalcode). This license permits unrestricted use, distribution, and reproduction in any medium, provided the original author and source are credited. 